\title{
COMPETENCIA PARA LA ORIENTACIÓN EDUCATIVA EN DOCENTES DE TECNOLOGÍA DE LA SALUD
}

Autor.

Yamilet Á. Seco

Profesor Titular de la Facultad de

Tecnología de la Salud, Cuba.

yavila@fts.scu.sld.cu
Coautor.

Belkis L. Aranda

Miembro del Centro de Estudios

Pedagógicos, Universidad de Oriente.

belkisac@uo.edu.cu
Coautor.

Irela M. Paz

Directora del Centro de

Estudios Pedagógicos, Universidad de Oriente.

Irela paz@uo.edu.cu

\section{País Origen: Ecuador}

Palabras claves: Formación, Competencia, Orientación, Tecnología, Salud.

Key Words: Formation, competition, orientation, technology, health.
Recibido: 11 de Marzo del 2016

Aceptado: 15 de Abril del 2016

\section{SUMMARY}

$\mathrm{T}$ his article starts from insufficiencies on professional development of professors at Health Technology Faculty, which limits their formative action towards their students. It is aimed to reveal some ideas for the formation of competition for educational orientation at Health Technology Faculty that contributes to the adequate professional development of professors as a via to improve the formative process of students. The formation of competition for educational orientation on the professors of this Faculty is elaborated on relationships and their basic dynamics is formed from the construction and welfare orientation management, through a process of reflection and pedagogic self reflection, assuming like a premise the pre-orientation formative awareness.

\section{RESUMEN}

E ste artículo parte de las insuficiencias en el desempeño profesional de los docentes de Tecnología de la Salud, que limitan su acción formativa hacia los estudiantes. Tiene como propósito revelar algunas ideas para la formación de la competencia para la orientación educativa en Tecnología de la Salud, que contribuya al adecuado desempeño profesional de los docentes como una vía para el mejoramiento del proceso formativo de los estudiantes. La formación de la competencia para la orientación educativa en los docentes de esta institución se conforma en las relaciones cuya dinámica fundamental se forma desde la constructividad y la gestión orientacional proasistencial, mediante un proceso de reflexión y autorreflexión pedagógica, asumiendo como premisa la concientización formativa preorientacional. 


\section{INTRODUCCIÓN}

$\mathrm{E}$

I perfeccionamiento continuo del Sistema Nacional de Educación establece, como objetivo esencial, la formación de una nueva generación, en correspondencia con las cualidades a que se aspira en este siglo XXI: formar de manera integral a niños, adolescentes y jóvenes.

La Educación Superior, como subsistema, tiene el propósito de lograr un profesional más pleno, creativo e independiente, dotado de cualidades de alto significado humano, capaz de poner sus conocimientos al servicio de la sociedad, lo cual implica prepararlo para asumir su autoeducación durante toda la vida, de modo que sea capaz de mantenerse actualizado, respondiendo a las necesidades del país, lo que ha generado que los centros universitarios estén en constante renovación y adquisición de saberes.

Se coincide con Pedro Horrutinier (2005), al considerar esencial la calidad de los docentes en estos centros, ya que contar con profesores preparados profesionalmente, con una adecuada formación pedagógica, así como consagrados plenamente a la labor educativa, es premisa para el logro de altos niveles de calidad.

En este sentido, en el país y en el territorio, se han realizado estudios sobre este tema, en los que se destacan especialistas como: Castellanos, N. (1999); García, G. y Addine, F. (1999); Valiente, P. (2001); Gato, C.A. (2003); Paz, I. (2005); Mediola, R. (2012), entre otros. Estos investigadores significan la importancia de una formación permanente orientada al perfeccionamiento de los educadores, considerada como un proceso sistemático y continuo, encaminada a la elevación creciente de su profesionalidad y a su crecimiento personal, para que sean capaces de transformar la realidad escolar por la vía científica, de manera que la experiencia acumulada se revierta en resultados cualitativamente superiores en el proceso formativo que dirigen.

Las investigaciones referidas se han centrado en los sujetos cuya formación específica ha sido como profesionales de la educación. Sin embargo, otros investigadores como Iglesia, N. (1999); Pupa, A. (2007); Díaz, M. (2010); García, I. (2011) manifiestan una profunda preocupación por la formación pedagógica de los docentes de Ciencias Médicas y, fundamentalmente, han profundizado en cómo favorecer la preparación de los docentes del área asistencial en contenidos didácticos.

Las exigencias de la universidad cubana actual requieren profundizar en la formación permanente de los docentes de las diferentes carreras de Ciencias Médicas, de manera que se contribuya a actualizar y ampliar en ellos sus conocimientos teóricos y metodológicos, además desarrollar competencias que le permitan el cumplimiento exitoso de la tarea instructiva y educativa que tienen asignada ante sus estudiantes.

En tal sentido, se reconoce la importancia de atender el perfeccionamiento constante de los docentes en las Facultades de Tecnología de la Salud, teniendo en cuenta su perfil de formación profesional; ya que una parte significativa de ellos no han sido graduados de carreras pedagógicas.

Profesionales como Vega, R. (2008); Díaz, M., Vidal, M. (2011) han estudiado la formación del docente de Tecnología de la Salud, haciendo énfasis en la preparación pedagógica, de manera que mejore su desempeño profesional.

La investigadora Travieso (2010), en sus indagaciones, establece la competencia del docente en Tecnología de la Salud, modelándola como integradora; sin embargo, no se deja ver de manera explícita lo relacionado con la función orientadora de este profesional como parte de su labor educativa.

La orientación educativa como parte de la función orientadora del docente, ha sido abordada por varios especialistas internacionales, nacionales y del territorio; estos reconocen la orientación educativa como un proceso de ayuda continua, preventiva y desarrolladora, dirigido a los sujetos en todos sus aspectos para resolver sus conflictos y necesidades; exponen vías y estrategias para movilizar los recursos personales y favorecer; por tanto, la formación integral de los estudiantes con ajuste a las características y necesidades de todos.

La generalidad de los mismos significa la necesidad de potenciar la función orientadora del docente; al respecto se coincide, ya que se considera limitado el despliegue de esta función por el profesional docente de Tecnología de la Salud.

La formación permanente de los profesores en las carreras de Tecnología de la Salud, en lo referido a la orientación educativa, adquiere gran significación, teniendo en cuenta que forman un profesional que tendrá la responsabilidad de brindar servicios de salud de calidad a la población. En tal sentido, los profesores de estas carreras deben estar preparados para proyectar y desarrollar acciones educativas que garanticen la orientación adecuada durante todo el proceso formativo de los estudiantes. 
A partir de esta situación se propone un modelo pedagógico de formación de la competencia para la orientación educativa en los docentes de Tecnología de la Salud, que contribuya al adecuado desempeño profesional de los docentes como una vía para el mejoramiento del proceso formativo de los estudiantes.

\section{DESARROLLO}

\section{Formación de la competencia para la orientación educativa en los docentes de Tecnología de la Salud.}

Esta competencia se forma en el proceso pedagógico de Tecnología de la Salud, en sus diferentes escenarios formativos como comités horizontales, colectivos de año, y en las diferentes vías de formación donde se insertan los docentes de Tecnología de la Salud, como el trabajo metodológico y la autopreparación. Una vía fundamental de formación de la competencia para la orientación educativa es a través del grupo de trabajo educativo, ya que da pautas para la proyección de acciones de orientación con vista a la prevención e intervención en los diferentes problemas y conflictos que puedan surgir en los estudiantes y grupos.

El proceso de formación de la competencia para la orientación educativa en los docentes de Tecnología de la Salud se concibe como un proceso de formación permanente, centrado en la autoeducación; transcurre en diferentes espacios y tiempos formativos en los que se insertan los docentes de Tecnología de la Salud, y se produce a través de las relaciones entre la constructividad en orientación proasistencial y la gestión orientacional proasistencial, mediante un amplio proceso de reflexión y autorreflexión pedagógica proorientacional.

Un aspecto direccional en este proceso de formación de la competencia para la orientación educativa lo constituye el elemento proasistencial que, como rasgo distintivo, se caracteriza por la preparación del docente en lo educativo formativo que permita potenciar en los estudiantes la retroalimentación de los contenidos básicos del tecnólogo, actitudes, sentimientos y pensamientos positivos los cuales definen la posición ético-afectiva de este ante la profesión para su desempeño en las unidades asistenciales de salud, con el objetivo de lograr la eficiencia de los servicios a partir de la correcta organización y ejecución del proceso tecnológico de la salud.

Este proceso, lo proasistencial implica tomar en cuenta que en la medida en que el docente de Tecnología de la Salud opera con herramientas apropiadas en la construcción del conocimiento del contenido de la profesión de las diferentes carreras, promueve en los estudiantes el desarrollo de estructuras lógicas y secuenciadas de este, que induzcan hacia el pensamiento activo y creativo, es decir, que les permita reflexionar, cuestionar, proponer, y solucionar problemas en torno a la profesión basado en las funciones básicas: asistencial, docente, investigativa y administrativa, además de desarrollar valores de humanismo, consagración, abnegación, liderazgo, prestigio, responsabilidad, profesionalidad, honestidad, desinterés, modestia, sentidos de la crítica y autocrítica, solidaridad e internacionalismo, que se reviertan en establecer relaciones de respeto a pacientes, familiares y equipo de trabajo en las unidades asistenciales de salud.

Para formar la competencia para la orientación educativa se toma lo asumido por Guillot, L. (2010) de Fuentes, H. (2006), que manifiesta la imperiosa necesidad de determinar los conocimientos, las habilidades y los valores que caracterizan a una competencia para su formación, se propone un acercamiento de los mismos en la competencia objeto de estudio:

Se constituyen en conocimientos básicos para esta competencia:

- Aspectos pedagógicos: el Modelo de formación en la universidad cubana actual y el Modelo de formación de los tecnólogos de la salud, los problemas profesionales de Tecnología de la Salud, tareas y funciones de los educadores, el diagnóstico como proceso, los proyectos educativos.

- $\quad$ Aspectos sociopsicológicos de la formación: las características biopsicosociales de los adolescentes y jóvenes universitarios, niveles y tareas del desarrollo de la edad, dominio de la comunicación asertiva, del trabajo grupal, la empatía, la tolerancia, la paciencia, el respeto a la diversidad y la concepción general de la orientación educativa.

\section{Como habilidades esenciales se significan:}

- Diagnosticar potencialidades y necesidades de los estudiantes de Tecnología de la Salud, para la proyección de acciones de orientación en los proyectos educativos de grupo y de año, con un enfoque humanista-desarrollador para garantizar su crecimiento personal en la solución de los problemas que permita los servicios de calidad en las unidades de salud.

- Elaborar el diagnóstico y pronóstico individuales y grupales para brindar atención especializada en correspondencia con el modelo de formación de las carreras de Tecnología de la Salud. 
- Comunicación adecuada que permita crear un clima de confianza, respeto, crítica constructiva y ayuda a la intervención en las problemáticas educativas, mostrando sus convicciones, sus actitudes y compromiso con la profesión.

- Determinar las acciones que se han de realizar en correspondencia con la determinación de las líneas de orientación.

- $\quad$ Evaluar el desarrollo alcanzado por los estudiantes y el grupo.

- Valorar críticamente su desempeño profesional para determinar las necesidades de su superación y el perfeccionamiento de su labor profesional como docente de Tecnología de la Salud.

Entre los valores se encuentran:

- La responsabilidad y laboriosidad con el nivel de preparación para cumplir con su papel profesional en la institución de formación de profesionales de la salud.

- Identidad profesional reflejada en el amor y actitud hacia la profesión, en el sentido y compromiso con la búsqueda de alternativas de solución a los problemas o conflictos que surjan en la marcha del proceso docente educativo de sus estudiantes, llevándolos a reflexionar sistemáticamente acerca de su desempeño en las áreas asistenciales de salud.

- $\quad$ El humanismo reflejado en la comprensión, sensibilización y discreción en la atención a los problemas de sus estudiantes.

Es premisa para la formación de esta competencia la concientización formativa preorientacional integrada por la identificación formativa especializada, la identificación formativa pedagógica y la audeterminación formativa para la orientación educativa en los docentes de Tecnología de la Salud.

El proceso de formación de la competencia para la orientación educativa en los docentes de Tecnología de la Salud se estructura a través de la constructividad orientacional proasistencial y la gestión orientacional proasistencia

I.

La constructividad orientacional proasistencial: consiste en la elaboración de sus propios conocimientos, del proyecto profesional por parte del docente de Tecnología de la Salud, asumiendo lo que necesita para dicha orientación (saberes, habilidades y valores), en función de garantizar servicios de calidad en las áreas asistenciales, a partir de: la habilitación orientacional, la disposición orientacional y la autoeducación en orientación educativa.

La habilitación orientacional: como elemento que dinamiza el proceso de formación permanente del docente de Tecnología de la Salud referido a la orientación educativa, se caracteriza por la búsqueda y adquisición del conocimiento, hábitos, habilidades y valores que necesita el docente de esta institución para desarrollar la orientación educativa en su desempeño profesional.

La disposición orientacional: es entendida como el proceso continuo, gradual, que el docente de Tecnología de la Salud lleva a cabo durante el proceso de formación permanente, despertando la actitud positiva y la motivación profesional para aprender de forma permanente a lo largo de la vida, facilitando la asimilación de los contenidos orientacionales y la adquisición de determinadas cualidades, sustentada en intereses y valores que le permiten actuar con iniciativa, perseverancia, reflexión en el proceso de autopreparación en herramientas necesarias para la correcta conducción de la orientación educativa hacia los estudiantes.

Estos dos componentes se sintetizan en la:

Autoeducación en orientación educativa: es el proceso que lleva a cabo el propio docente de Tecnología de la Salud en su preparación y autopreparación en conocimientos, habilidades y dispuestos para realizar con éxito la orientación educativa, implica la formación de su personalidad, su preparación para insertarse en la labor orientadora. De la relación entre los componentes del subsistema se revela la función: apropiación del contenido de orientación.

La Gestión orientacional proasistencial: es vista como el instrumento esencial que permite organizar, planificar, desarrollar y controlar la formación de los docentes de Tecnología de la Salud, tanto individual como grupal, lo que permite ir a la búsqueda de nuevos conocimientos, análisis, valoraciones acerca de su quehacer en la formación de un futuro tecnólogo con vocación y ética profesional, así como valores morales y humanos que le permitan interactuar con el equipo de salud, el colectivo de trabajo, los pacientes, la familia y la comunidad.

Para esta gerstión se tienen en cuenta los siguientes componentes: la identificación de necesidades, la proyección orientadora y la actuación orientacional. 


\section{Estos componentes se sintetizan en la:}

Actuación orientacional: mediante esta el docente de Tecnología de la Salud pone en práctica la estrategia elaborada y su evaluación, a través de la aplicación de la estrategia, su valoración y/o reajuste.

De la relación entre los componentes del subsistema se revela la función de despliegue del proceso de orientación.

Las funciones que surgen de los subsistemas se relacionan dialécticamente ya que la competencia para la orientación educativa se muestra a través del despliegue del proceso de orientación, de acuerdo a la apropiación que tenga el docente del contenido de orientación, la formación permanente es un elemento dinamizador de esta relación que influye en la identificación de las contradicciones que surgen en el proceso. La relación entre estas funciones es la vía para poder concretar la competencia para la orientación educativa en los docentes de Tecnología de la Salud.

La construcción orientacional y la gestión orientacional, se caracterizan por tener funciones distintas, se desarrollan a la vez e interrelacionan dialécticamente revelándose una regularidad: la formación - autoformación para la orientación educativa en los docentes de Tecnología de la Salud.

La formación-autoformación para la orientación educativa en el docente significa integrar el modo de concebir y asimilar todo lo que viene del conocimiento, de la experiencia y del sentimiento, ya sea transferido o autogestionado mediante la relación entre la construcción y la gestión para realizar la orientación educativa, tomando como premisa la concientización para esta orientación. Esta premisa de concientización formativa preorientacional del docente parte de que él mismo identifica y toma conciencia de sus propias necesidades de autopreparación para la realización de la orientación educativa, proyecta sus metas y vías de transformación.

La constructividad orientacional implica la apropiación del conocimiento, habilidades, hábitos y valores que necesita el docente para ese desempeño profesional a través de diferentes vías y espacios, dirigidos por el coordinador del colectivo de año y el jefe de grupo de trabajo educativo, lo que permite la apropiación de lo relacionado con la actividad realizada, estimulando una motivación profesional en el logro de los objetivos trazados, y que sienta la necesidad de aprender esos conocimientos y habilidades para alcanzarlos, sustentado en intereses por el proceso mismo de asimilación, de manera que lo sienta como algo atractivo y grato, con compromiso, dándole significado y sentido a lo que asimila, lo cual se expresa en el rol de ser educador.

La gestión orientacional significa que, a partir de la identificación de las carencias de orientación en los estudiantes, se planea acciones y actividades de orientación; de acuerdo con el resultado alcanzado en su desempeño, se autovalora y reflexiona en cuanto a la identificación de esas nuevas necesidades para realizar la orientación educativa en los estudiantes. En la medida en que el docente se conozca a sí mismo y valore de forma adecuada los distintos contenidos y funciones que debe dominar en relación con la labor orientadora, puede trazarse objetivos y metas que comprendan la proyección futura y una aspiración acorde con sus posibilidades reales, así como realizar esfuerzos conscientes para alcanzar sus objetivos.

\section{CONCLUSIONES}

La dinámica del proceso de formación de la competencia para la orientación educativa en los docentes de Tecnología de la Salud descansa en la relación que se establece entre las funciones de la construcción y la gestión orientacional proasistencial, mediante un proceso de reflexión y autorreflexión pedagógica, asumiendo como premisa la concientización formativa preorientacional, para lograr un proceso formativo de este docente a la altura de las nuevas exigencias de desempeño que requiere este profesional. 


\section{BIBLIOGRAFÍA}

Addine, F. (2006). Modos de actuación profesional pedagógica. De la teoría a la práctica. La Habana: Editorial, Academia.

Castellanos Fuentes. N. (1999). La formación de los profesores de Química en la provincia de Pinar del Río, Tesis en opción del título de Máster en Ciencias de la Educación, Pinar del Rio.

Díaz Rivero, M. (2010). Desafíos y respuestas para el fortalecimiento de los valores en la Salud Pública en Cuba. Recuperado el 19 de noviembre de 2013, de http://scielo.sld.cu/scielo.php

García Álvarez, I. (2011). La labor formativa del profesor guía de la carrera de Medicina en el modelo policlínico universitario. Tesis doctoral en Ciencias Pedagógicas, UCP "Frank País García". Santiago de Cuba.

Gato Armas, C. A. (2003). La formación permanente de profesores de mecánica en centros politécnicos industriales y escuelas de oficios. Tesis en opción al grado académico de Máster en Pedagogía Profesional, Pinar del Río.

Horruitiner Silva, P. (2005). Fundamentos del proceso de formación en la Educación Superior, La Habana.

Iglesis Morell, N. (2008). Preparación psicopedagógica del docente asistencial de Licenciatura en Enfermería. Propuesta de un sistema de superación, Tesis en opción al título de Master en Investigación Educativa, (Versión Digital) Camaguey.

Mediola González, R. (2012). Estrategia para la formación pedagógica ambiental de los profesores asesores de Misión Sucre. Tesis doctoral en Ciencias Pedagógicas, Santiago de Cuba.

Paz Domínguez, I. M. (2005). El colectivo de año en la orientación educativa a los estudiantes de las carreras pedagógicas. Tesis doctoral en Ciencias Pedagógicas. Instituto Superior Pedagógico "Frank País García", Santiago de Cuba.

Puga García, A. y Madiedo Albolatrach, M. (2007). Consideraciones sobre la clase taller en la formación del profesional en Ciencias Médicas. Recuperado el 19 de noviembre del 2013, de http://scielo.sld.cu/scielo.php

Travieso, N. (2010). Alternativa para el desarrollo de competencias profesionales en la superación del docente de tecnología de la salud. Tesis doctoral en Ciencias Pedagógicas, Universidad de Ciencias Pedagógicas "Frank País García", Santiago de Cuba.

Vidal Ledo, M., Hernández García, L. (2011). Superación profesoral. Educación Médica Superior, 25(2):206-216. Recuperado en mayo del 2013, de http://bvs.sld.cu/revistas/ems/vol20_03_06/ems12306.htm 